
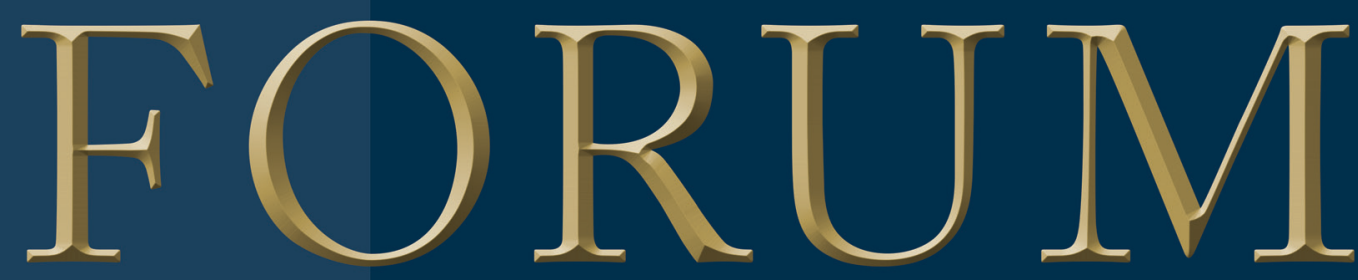

VOLUME 28 | NUMBER 2 MARCH/APRIL 2018

HAIR TRANSPLANT FORUM INTERNATIONAL

IN THIS ISSUE

The Evolving Role for Autologous Adipose

Tissue (Fat) in Treating Hair Loss

A Case Report of Subclinical Lichen Planopilaris after Hair Transplantation

Spotlight on Ethical Marketing

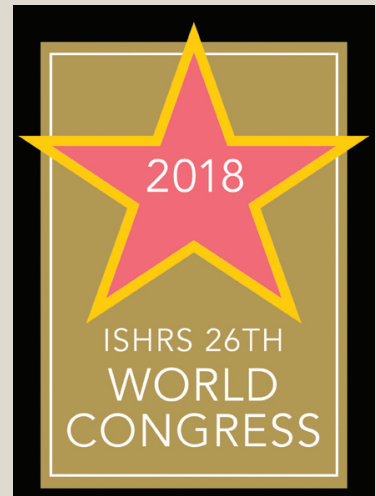

OCTOBER 10-14 HOLLYWOOD LOEWS HOLLYWOOD HOTEL www.ISHRS.org

\section{Graft Quality Index: A Morphologic Classification of Follicular Unit Excision (FUE) Grafts}

\author{
Robert H. True, MD, MPH, FISHRS I New York, New York, USA I drtrue@hairlossdoctors.com
}

\section{GRAFT QUALITY INDEX}

Graft quality is a significant component of all hair restoration surgery, especially Follicular Unit Excision (FUE). There is much discussion in our professional community about the impact of graft quality on the cosmetic results of hair restoration surgery, particularly with FUE. ${ }^{1-4}$ The gold standard remains that microscopically slivered and created grafts obtained by strip surgery are ideal. The challenge for FUE harvesting methods is to produce grafts that are similar to or exactly the same as strip grafts. FUE grafts are often characterized as having lower yield than microscopically dissected grafts produced in FUT surgery.

Grafts of high quality, whether produced by strip or FUE, have supportive tissue throughout and contain minimal transections, follicle fractures, and crushed follicles. Transection and stripped and severely splayed follicles are by-products of the punch insertion technique. Follicle fractures and crush injury are consequences of the amount and type of force used to remove the grafts once they have been scored and dissected.

The gold standard remains that microscopically slivered and created grafts obtained by strip surgery are ideal. The challenge for FUE harvesting methods is to produce grafts that are similar to or exactly the same as strip grafts.

Grafts of high quality are amenable to placement without undue manipulation or placement trauma. Grafts of lesser quality present more risk of damage during processing and implantation. In inspecting FUE grafts produced by a wide variety of techniques, one can observe that the grafts have different morphologies. On closer analysis, FUE grafts fall into four morphological types. Although there is general consensus in our field that some graft morphologies produce better yields than others, we do not know what the impact of these different morphologies is on transplant outcomes.

These morphological types are the basis of my novel concept, Graft Quality Index (GQI). I propose that this index can be used in all hair restoration surgeries-including FUE- to grade grafts. The graft quality grade can be used as a quality control tool to 1) predict the difficulty of graft placement, 2) guide the best implantation technique, and 3) relate graft morphology to the results of surgery. Perhaps this will help to answer the question of the effect on graft morphology on outcomes in future studies.

GQI has four grades:

1. Grade 1: Grafts have no transections or damaged follicles, a smooth regular border, perifollicular tissue throughout the follicle length, and nonfollicular tissue below the bulbs (Figure 1). 
FIGURE 2. GQI Grade 2 grafts

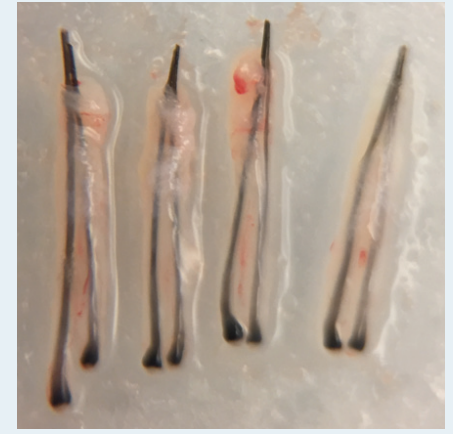

FIGURE 3. GQI Grade 3 grafts
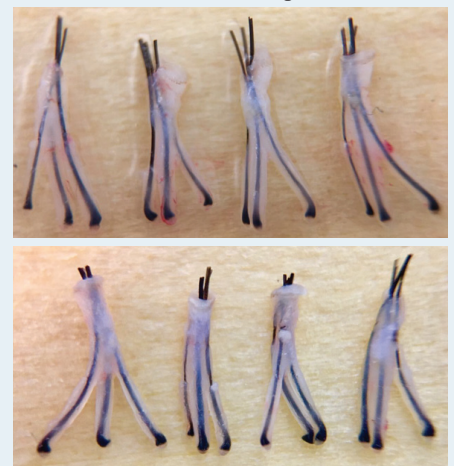

2. Grade 2: Grafts are similar to those in Grade I but have less perifollicular tissue and no tissue below the bulbs. They also are free of transections and follicle damage (Figure 2).

3. Grade 3: Grafts have extreme iatrogenic splay, with the lower third to half of the follicles being denuded of surrounding tissue (Figure 3). The concept of iatrogenic splay will be explained below.

4. Grade 4: Grafts contain transections and damaged follicles, the graft margins are irregular and transected follicles may protrude from the graft, and some follicles may be denuded and have iatrogenic splay (Figure 4).

FIGURE 4. GQI Grade 4 grafts

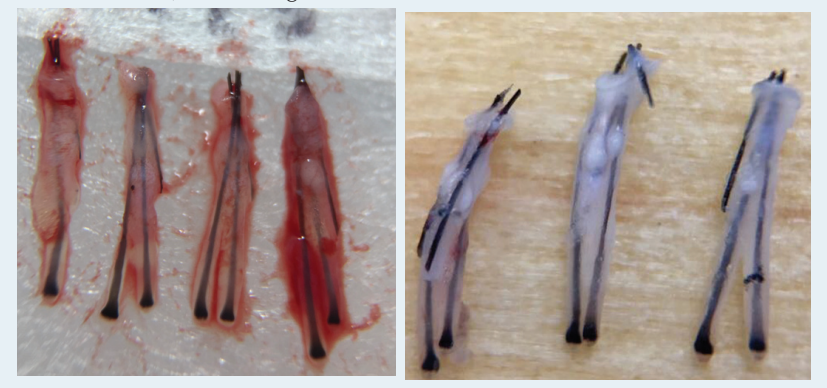

The distinguishing characteristics of the grafts in each GQI class are summarized in Table 1. Table 2 is an example of how to score a case with GQI. To calculate the GQI score, the following method is suggested: one point is assigned for each Grade 1 graft, 2 points assigned for each Grade 2 graft, 3 points for Grade 3 grafts, and 4 points for Grade 4 grafts. The point total is divided by the graft total to get the GQI score. Lower scores indicate more grafts with the most favorable characteristics: easiest to place and more likely to survive. Higher scores above 2 imply more grafts with less

TABLE 1. Distinguishing Characteristics of Grafts in GQI Classes

\begin{tabular}{|c|c|c|c|c|c|c|}
\hline GQI Class & Splay & Transection & $\begin{array}{l}\text { Denuded } \\
\text { Follicles }\end{array}$ & $\begin{array}{c}\text { Graft } \\
\text { Margins }\end{array}$ & $\begin{array}{l}\text { Supportive } \\
\text { Tissue }\end{array}$ & $\begin{array}{l}\text { Tissue } \\
\text { Below } \\
\text { Bulbs }\end{array}$ \\
\hline 1 & $\begin{array}{l}\text { Structural } \\
\text { Only }\end{array}$ & None & None & $\begin{array}{l}\text { Smooth } \\
\text { and regular }\end{array}$ & Abundant & Yes \\
\hline 2 & $\begin{array}{l}\text { Structural } \\
\text { Only }\end{array}$ & None & None & $\begin{array}{l}\text { Smooth } \\
\text { and } \\
\text { Regular }\end{array}$ & Scant & No \\
\hline 3 & $\begin{array}{l}\text { Extreme } \\
\text { latrogenic }\end{array}$ & Some & Common & $\begin{array}{c}\text { Bare } \\
\text { Follicles }\end{array}$ & No & No \\
\hline 4 & $\begin{array}{l}\text { Some } \\
\text { latrogenic }\end{array}$ & Common & Occasional & $\begin{array}{l}\text { Irregular } \\
\text { with } \\
\text { Protruding } \\
\text { Transected } \\
\text { Follicles }\end{array}$ & Irregular & No \\
\hline
\end{tabular}

TABLE 2. Scoring with GQI

\begin{tabular}{|c|l|l|l|}
\hline GQI Graft Grade & Graft Number & Point Count & \\
\hline 1 & 1250 & 1250 & \\
\hline 2 & 250 & 500 & \\
\hline 3 & 95 & 285 & \\
\hline 4 & 45 & 180 & GQI Score 1.35 \\
\hline Total & 2640 & 2215 &
\end{tabular}

favorable characteristics: more difficult to place without risk of trauma and more likely to have lower graft survival rates.

To illustrate the application of GQI, I will give two examples from the Graft Analysis Project conducted as part of the ISHRS Live Surgery Workshop in Polanica, Poland, in 2017.

The first sample, shown in Figure 5, had the lowest GQI score among the workshop cases, and the second sample, shown in Figure 6, had the highest GQI score among the cases. In the lowest score case, the majority of grafts fell into GQI Grade 1 and the transection rate was low suggesting high graft quality and the likelihood of a good outcome. In the case with the highest score, all of the grafts were either denuded or contained follicle damage indicating poor graft quality and probability of a lesser outcome.
FIGURE 5. Case with low GQI score

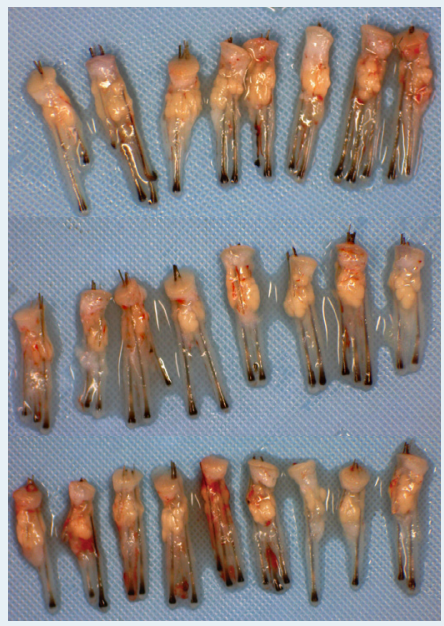

Punch diameter at the tip 1.05 Blunt Punch

Grade 1 - 19; Grade 2 - 1;

Grade 3 - 0; Grade 4 - 5

Total points -41

GQI - 1.64

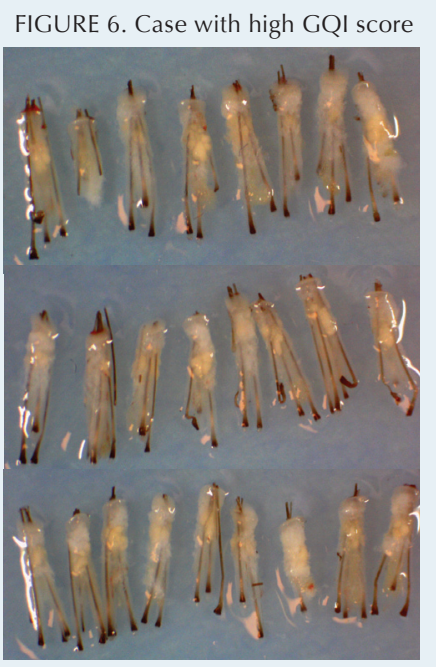

0.87 outside diameter

Sharp Motorized

Grade 1 - 0; Grade 2 - 0;

Grade 3 - 12; Grade 4 - 13

Total points -88

GQI -3.52
I recommend that GQI should be recorded as 1) punch diameter, 2) punch type, 3) count per Grade, 4) total points, and 5) GQI score. All of this information will be needed to interpret the meaning of the score.

The character of GQI class 3 grafts must be elaborated further. In the standard terminology of FUE (Figure 7), splay is the term used to describe a follicle (or all follicles) within a follicular cluster that diverge from adjacent follicles. ${ }^{5-7}$

Splay is typically observed in the proximal portion (lower one-third) of the follicular unit,

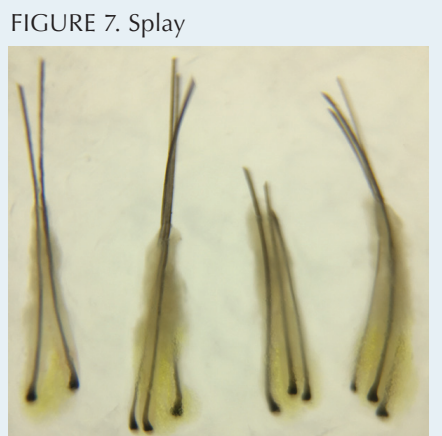


and it can vary in degree. I want to introduce the concept of "structural" splay and "iatrogenic" splay. Structural splay exists anatomically in the tissue and varies among patients, and importantly varies within the same patient (Figure 8). latrogenic splay is defined as follicular splay that is produced by the method of FUE harvesting.

FIGURE 8. Variable structural splay
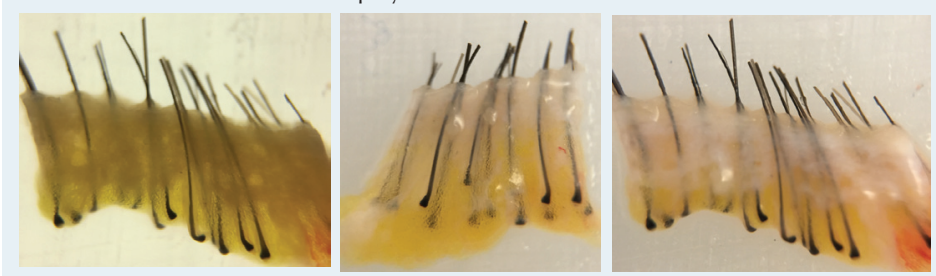

FUE techniques in which the punch is inserted superficially into the dermis may often produce extreme splay of the bulb portion of the follicles. This is a consequence of stripping away the perifollicular tissue during the

FIGURE 9. latrogenic splay

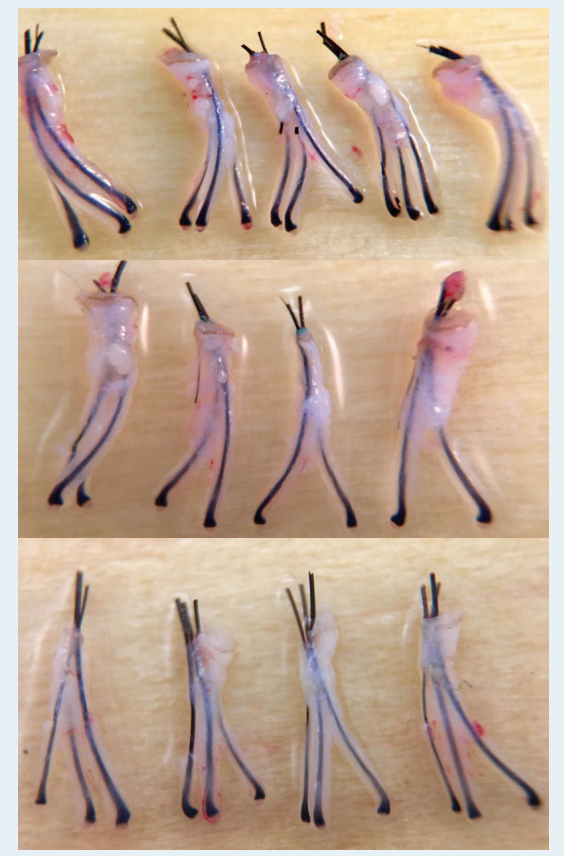

extraction phase of follicular unit excision (Figure 9). Such grafts are more difficult to place without additional trauma and are more amenable to implanter placement. Graft quality problems such as skeletonization typically accompany iatrogenic splay. Sometimes with sharp punches it is difficult to find the exact punch insertion depth that eliminates both transections and iatrogenic splay. This is particularly true for novice surgeons but also can be a challenge in some cases

FIGURE 10. Crushed bulb

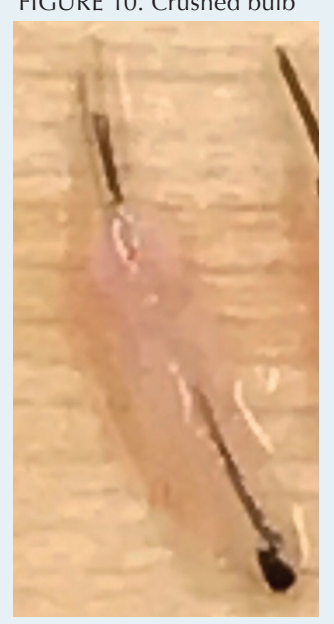

even for the most experienced surgeons. Dull and hybrid punches can usually be inserted deeper without increasing transections and tend to reduce iatrogenic splay producing the highest percentage of GPI Grade 1 and 2 grafts.

Grade 1 and 2 grafts are of the highest quality and are easy to place without damage by skilled use of forceps. Grades 3 and 4 grafts are of lesser quality and are more difficult to place without damage with forceps. When the grafts have little if any perifollicular tissue, they must be handled very delicately. It is possible to crush the bulbs and/or fracture the follicles even with light forceps pressure (Figure 10). When placing splayed follicles with forceps, they must be gathered gently together. If one or more follicles is not contained within the forceps, they may "catch" or be traumatized on the surface of the skin during placement. This may prevent complete insertion, resulting in the risk of additional trauma as the graft may have to be placed again (Figure 11). As a consequence, there is an increased risk of Repetitive Placement Trauma (RPT), as described by Dr. Wolf, to the graft. ${ }^{8} \mathrm{~A}$ similar problem in graft placement arises when the graft contains transected follicle shafts protruding from its surface. These grafts also have less risk of injury when placed with implanters. The skeletonized, splayed, and transected follicles can be gathered without touching within the implanter and inserted in a single step (Figure 12).

\section{What sample size should be used in applying GQI?}

While all grafts in a case could be scored according to GQI, I believe it is possible to establish a reliable score by selecting a representative random sample of the grafts. I think a $10 \%$ sample is adequate. So, for a 2,000-graft case, 200 grafts would need to be scored. To obtain a representative sample, the selected grafts should be based on the proportion of each graft size obtained. In the 2,000-graft case example, let's say $20 \%$ are singles, 55\% are doubles, $20 \%$ triples, and 5\% quadruples. Thus, for the 200-graft sample, 40 would be singles, 110 doubles, 40 triples, and 10 quadruples. All of the grafts of each size should be mixed together, and the required number of sample grafts selected from different parts of the pile after mixing the grafts with forceps between selections. In order to minimize selection bias, the grafts should be selected with a naked eye and without trying to look at the detail of the graft. While such an approach is not rigorously scientific, I believe it is a practical approach to GQI grading that can be easily conducted without adding significantly to the time needed to perform the case.

\section{How does GQI apply to strip harvest procedures?}

The Graft Quality Index can also apply to grafts produced 
by microscopic dissection with strip harvest procedures, but with some modification in explanation. Type $3 \mathrm{GQI}$ grafts characterized by iatrogenic splay and denuded follicles are unique to FUE surgery and unlikely to be produced with microscopic graft preparation. With FUT, Type $1 \mathrm{GQI}$ grafts are produced when FUT dissection style is to produce "chubby" grafts. Type $2 \mathrm{GQI}$ grafts are typical of FUT dissection style that produces "skinny" grafts. Type $3 \mathrm{GQI}$ grafts would be unlikely with microscope dissection, but theoretically could occur if grafts are over-dissected producing denuded follicles. Type $4 \mathrm{GQI}$ grafts occur with strip surgery secondary to follicle damage during strip harvest or microscopic dissection. Applying GQI to FUT procedures could still give useful information in that it would characterize the type of grafts being produced and the number of grafts with damaged follicles.

\section{LIMITATIONS OF GQI}

Graft Quality Index is applied only to grafts deemed available and suitable for transplantation. Excluded from GQI

TABLE 3. Quality Control in FUE

\begin{tabular}{|c|l|}
\hline \multicolumn{2}{|c|}{ Comprehensive FUE Quality Control } \\
\hline Measurements & Donor Density \\
& Punch insertions \\
& Capped grafts \\
& Pared grafts \\
& Broken grafts \\
& Partially transected grafts \\
& Totally Transected grafts \\
& Buried grafts \\
& Missing grafts \\
& Grafts available for \\
& transplantation \\
& Grafts unavailable for \\
& transplantation \\
\hline Calculations and Rates & Missing graft rate \\
& Follicle transection rate \\
& Graft transection rate \\
& Average hairs per graft \\
& Calculated Follicles per graft \\
& expected and achieved \\
\hline Graft Morphology & Graft Quality Index \\
\hline
\end{tabular}

\section{CONCLUSION}

FUE grafts fall into four basic morphological types. These types are the basis of the GQI. FUE grafts can be graded according to the GQI in order to evaluate the quality of the grafts in relation to the excision process, to tailor placement technique for each type of graft, and as a Quality Control measure in relationship to surgery outcomes. GQI is also applicable to strip harvest procedures.

\section{References}

1. Beehner, M.L. Comparison of survival of FU grafts trimmed chubby, medium and skeletonized. Hair Transplant Forum Int'l. 2010; 20(1):1,6.

2. Harris, J.A. Follicular Unit Extraction. Facial Plast Surg Clin North Am. 2013(Aug); 21(3):375-84. PMID: 24017979. doi: 10.1016/j. fsc.2013.05.002.

3. Cole, J.P. Status of individual follicular group harvesting. Hair Transplant Forum Int'l. 2009; 19(1):20-24.

4. Crisostomo, M. Comparison of survival in FUE vs FUT grafts. Italian society of Hair Science and Surgery, Venice Italy, May 2017.

5. Lorenzo, J. Standardization of the terminology used in FUE: Part I. Hair Transplant Forum Int'l. 2013; 23(5):165-168.

6. Cole, J. Standardization of the terminology used in FUE: Part II. Hair Transplant Forum Int'l. 2013; 23(6):210-212.

7. Cole, J. Standardization of the terminology used in FUE: Part III. Hair Transplant Forum Int'l. 2014; 24(3):93-94.

8. Wolf, B.R. The art and craft of recipient site creation and graft placement. Hair Transplant Forum Int'l. 2014; 24(2):41,46-49.
The best practice is to routinely monitor and count key quality indicators in all surgeries. These are summarized in Table 3 and are explained in detail in the ISHRS Standard Terminology of
FUE. ${ }^{6,7,8}$ are missing grafts, and grafts with Therefore, GQI is not sufficient as the only quality control measure in FUE.

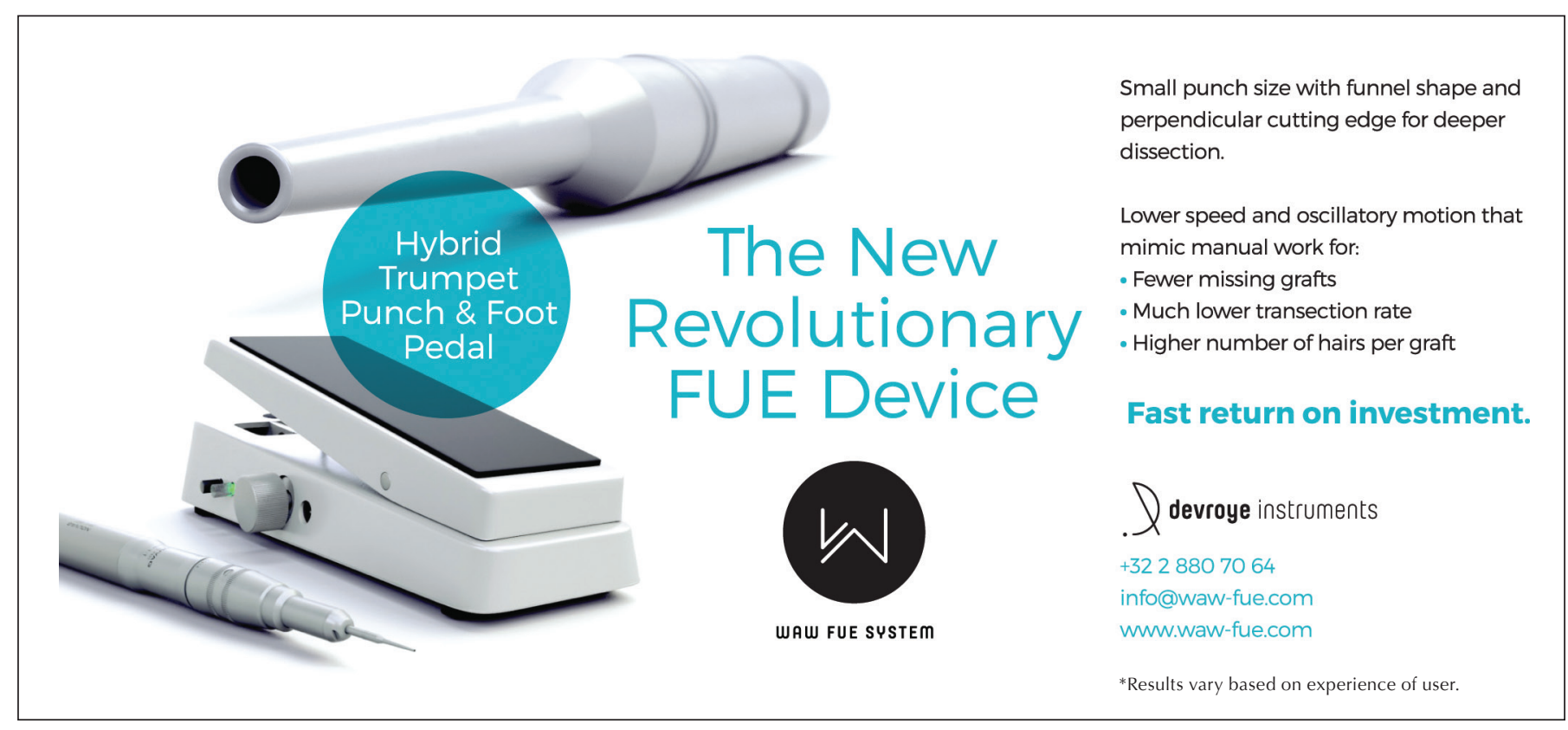

